\title{
A New Class of Chiral Pyrrolidine-Pyridine Conjugate Base Catalysts for Use in Asymmetric Michael Addition Reactions
}

Takaaki Ishii, Shingo Fujioka, Yusuke Sekiguchi, and Hiyoshizo Kotsuki*

Laboratory of Natural Products Synthesis, Faculty of Science, Kochi University, Akebono-cho, Kochi 780-8520, Japan kotsuki@cc.kochi-u.ac.jp

Experimental Procedures

(9 Pages) 


\section{Materials.}

2-Bromo-4-dimethylaminopyridine was prepared according to the literature procedure. $^{1}$ 2-Bromo-4-pyrrolidinopyridine was also prepared in a very similar manner as follows. 1,2Dibromotetrafluoroethane was purchased from TCI and used as received.

\section{2-Bromo-4-pyrrolidinopyridine.}<smiles>c1cc(N2CCCC2)ccn1</smiles>

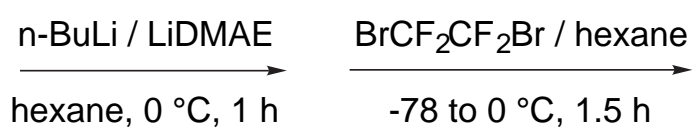<smiles>Brc1cc(N2CCCC2)ccn1</smiles>

A solution of 2-(dimethylamino)ethanol (DMAE) $(2.41 \mathrm{~mL}, 24$ mmol) in hexane $(30 \mathrm{~mL})$ was cooled at $0{ }^{\circ} \mathrm{C}$, and $\mathrm{n}$-BuLi $(30.4 \mathrm{~mL}, 48$ mmol) was added dropwise under Ar. After $30 \mathrm{~min}$ at this temperature, 4-pyrrolidinopyridine $(1.78 \mathrm{~g}, 12 \mathrm{mmol})$ was added as a solid. After $1 \mathrm{~h}$ at $0{ }^{\circ} \mathrm{C}$, this orange colored mixture was recooled to $-78{ }^{\circ} \mathrm{C}$, and a solution of 1,2-dibromotetrafluoroethane $(3.64 \mathrm{~mL}, 30 \mathrm{mmol})$ was added dropwise. After $30 \mathrm{~min}$ of stirring at $-78^{\circ} \mathrm{C}$, the mixture was allowed to warm to $0{ }^{\circ} \mathrm{C}(1.5 \mathrm{~h})$. Hydrolysis was performed at this temperature with $\mathrm{H}_{2} \mathrm{O}$. The aqueous phase was first extracted with diethyl ether and then with dichloromethane. The extracts were dried $\left(\mathrm{MgSO}_{4}\right)$, evaporated, and purified by silica gel column chromatography (hexane / AcOEt = 1:1) to give 2-bromo-4-pyrrolidinopyridine $(1.23 \mathrm{~g}, 45 \%)$ as a colorless solid.

M.p. 138.5-139.5 ${ }^{\circ} \mathrm{C}$ (recryst from $\mathrm{Et}_{2} \mathrm{O}$-hexane).

FTIR $(\mathrm{KBr}) \vee 1619,1594,1505,1249,1122,1071,989,971,810 \mathrm{~cm}^{-1}$.

${ }^{1} \mathrm{H}$ NMR (400 MHz, $\left.\mathrm{CDCl}_{3}\right) \delta 2.03(4 \mathrm{H}, \mathrm{m}), 3.28(4 \mathrm{H}, \mathrm{m}), 6.31(1 \mathrm{H}, \mathrm{d}, J$ $=5.2 \mathrm{~Hz}), 6.51(1 \mathrm{H}, \mathrm{s}), 7.90(1 \mathrm{H}, \mathrm{d}, J=5.2 \mathrm{~Hz})$.

${ }^{13} \mathrm{C}$ NMR $\left(100 \mathrm{MHz}, \mathrm{CDCl}_{3}\right) \delta 25.19(\times 2), 47.11(\times 2), 106.63,109.34$, $142.74,149.09,153.16$.

Anal. Calcd for $\mathrm{C}_{9} \mathrm{H}_{11} \mathrm{BrN}_{2}$ : C, 47.60; H, 4.88; N, 12.34. Found: C, 
$47.75 ; \mathrm{H}, 4.59 ; \mathrm{N}, 12.02$.

\section{General Procedure for the Preparation of Chiral Pyrrolidine- Pyridine Conjugate Base Catalysts.}

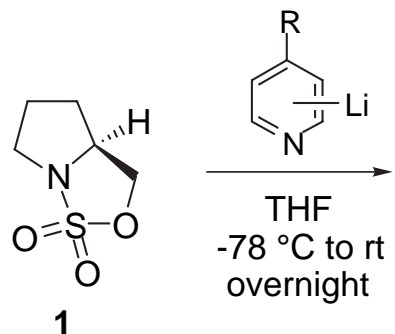<smiles>[R]c1ccnc(CC2CCCN2S(=O)(=O)O)c1</smiles><smiles>CO[C@H](OCO)[C@H](OC)OCO</smiles>
overnight

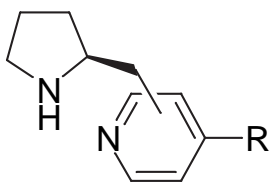

$3(50-87 \%)$

To a solution of the bromopyridine derivative $(1.05 \mathrm{~mL}, 11 \mathrm{mmol})$ in dry THF (12 mL) at $-78{ }^{\circ} \mathrm{C}$ was added $1.59 \mathrm{M} n$-BuLi in hexane $(7 \mathrm{~mL}$, $11 \mathrm{mmol}$ ) and the mixture was stirred at this temperature for $1 \mathrm{~h}$. Then to this solution was added at $-78{ }^{\circ} \mathrm{C}$ a solution of cyclic sulfamate $\mathbf{1}$ $(1.37 \mathrm{~g}, 8.4 \mathrm{mmol})$ in dry THF $(10 \mathrm{~mL})$ and the mixture was allowed to warm to $\mathrm{rt}(12 \mathrm{~h})$. After evaporation of the solvent, the resulting beige foam was treated with hot $16 \mathrm{~mL}$ of $2 \mathrm{~N} \mathrm{HCl}$ and $16 \mathrm{~mL}$ of EtOH (overnight). The mixture was basified with $50 \% \mathrm{NaOH}$ solution, and extracted with $\mathrm{CH}_{2} \mathrm{Cl}_{2}$. The extracts were dried $\left(\mathrm{MgSO}_{4}\right)$, evaporated, and purified by silica gel column chromatography $\left(\mathrm{CHCl}_{3} / i-\mathrm{PrNH}_{2}=9\right.$ :

1) to give the desired pyrrolidine-pyridine conjugate base catalyst.

(S)-2-(Pyrrolidin-2-ylmethyl)pyridine (3a).

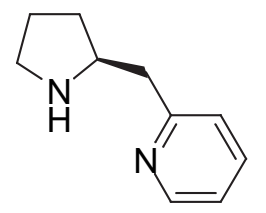

$53 \%$ yield, pale yellow oil; $\mathrm{R}_{\mathrm{f}} 0.27\left(\mathrm{CHCl}_{3} / i-\mathrm{PrNH}_{2}=19: 1\right)$.

$[\alpha]_{\mathrm{D}}^{24}+15.5(c 0.90, \mathrm{MeOH})$.

FTIR (neat) $v 3284,1592,1568,1474,1436,753 \mathrm{~cm}^{-1}$.

${ }^{1} \mathrm{H} \mathrm{NMR}\left(400 \mathrm{MHz}, \mathrm{CDCl}_{3}\right) \delta 1.45(1 \mathrm{H}, \mathrm{ddt}, J=12.2,9.3,7.8 \mathrm{~Hz})$, 1.70-1.95 $(3 \mathrm{H}, \mathrm{m}), 2.76(1 \mathrm{H}, \mathrm{br}), 2.85-2.92(1 \mathrm{H}, \mathrm{m}), 2.89(1 \mathrm{H}, \mathrm{dd}, J=$ $13.5,7.8 \mathrm{~Hz}), 2.98(1 \mathrm{H}, \mathrm{dd}, J=13.5,5.6 \mathrm{~Hz}), 3.05(1 \mathrm{H}, \mathrm{ddd}, J=10.2$, 
7.6, $5.4 \mathrm{~Hz}), 3.51(1 \mathrm{H}, \mathrm{tt}, J=7.8,5.6 \mathrm{~Hz}), 7.12(1 \mathrm{H}, \mathrm{ddd}, J=7.8,4.9$, $1.2 \mathrm{~Hz}), 7.19(1 \mathrm{H}, \mathrm{d}, J=7.8 \mathrm{~Hz}), 7.60(1 \mathrm{H}, \mathrm{dt}, J=7.8,2.0 \mathrm{~Hz}), 8.53(1 \mathrm{H}$, $\mathrm{ddd}, J=4.9,2.0,1.0 \mathrm{~Hz})$.

${ }^{13} \mathrm{C}$ NMR $\left(100 \mathrm{MHz}, \mathrm{CDCl}_{3}\right) \delta 24.96,31.23,44.12,46.17,58.90,121.22$, $123.53,136.33,149.24,160.11$.

MS $m / z$ (rel intensity) $161\left(\mathrm{M}^{+}-1,2\right), 133$ (2), 106 (5), 93 (52), 70 (100), $65(7)$.

HRMS calcd for $\mathrm{C}_{10} \mathrm{H}_{14} \mathrm{~N}_{2}-\mathrm{H} 161.1079$, found 161.1059 .

(S)-4-Dimethylamino-2-(pyrrolidin-2-ylmethyl)pyridine $(3 \mathrm{~b})$.

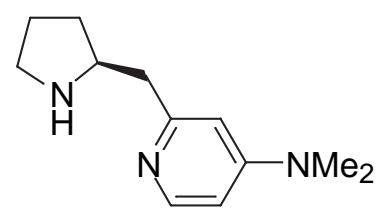

$80 \%$ yield; pale yellow oil; $\mathrm{R}_{\mathrm{f}} 0.19\left(\mathrm{CHCl}_{3} / i-\mathrm{PrNH}_{2}=9: 1\right)$.

$[\alpha]_{\mathrm{D}}^{22}-7.9\left(c 0.76, \mathrm{CHCl}_{3}\right)$.

FTIR (neat) v 3327, 1602, 1542, 1507, 1374, 995, 805, $750 \mathrm{~cm}^{-1}$.

${ }^{1} \mathrm{H}$ NMR $\left(400 \mathrm{MHz}, \mathrm{CDCl}_{3}\right) \delta 1.43(1 \mathrm{H}, \mathrm{ddt}, J=12.0,9.3,7.6 \mathrm{~Hz})$, 1.67-1.93 (3H, m), $2.47(1 \mathrm{H}, \mathrm{br}), 2.74(1 \mathrm{H}, \mathrm{dd}, \mathrm{J}=13.2,8.0 \mathrm{~Hz}), 2.81$ $2.86(1 \mathrm{H}, \mathrm{m}), 2.85(1 \mathrm{H}, \mathrm{dd}, J=13.2,5.6 \mathrm{~Hz}), 2.98(6 \mathrm{H}, \mathrm{s}), 2.95-3.06(1 \mathrm{H}$, $\mathrm{m}), 3.46(1 \mathrm{H}$, quintet, $J=6.6 \mathrm{~Hz}), 6.35(1 \mathrm{H}, \mathrm{dd}, J=6.1,2.7 \mathrm{~Hz}), 6.40$ $(1 \mathrm{H}, \mathrm{d}, J=2.7 \mathrm{~Hz}), 8.15(1 \mathrm{H}, \mathrm{d}, J=6.1 \mathrm{~Hz})$.

${ }^{13} \mathrm{C} \operatorname{NMR}\left(100 \mathrm{MHz}, \mathrm{CDCl}_{3}\right) \delta 24.82,31.18,39.02(\times 2), 44.80,46.11$, $58.92,104.53,105.93,149.19,154.71,159.98$.

MS m/z (rel intensity) $205\left(\mathrm{M}^{+}, 54\right), 204$ (60), 176 (23), 163 (32), 149 (20), 136 (100), 121 (42), 93 (15), 80 (13), 70 (80), 68 (13).

HRMS calcd for $\mathrm{C}_{12} \mathrm{H}_{19} \mathrm{~N}_{3} 205.1579$, found 205.1572

(S)-4-Pyrrolidino-2-(pyrrolidin-2-ylmethyl)pyridine (3c).

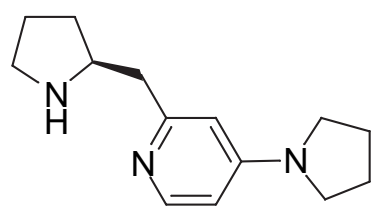


$50 \%$ yield; pale yellow oil; $\mathrm{R}_{\mathrm{f}} 0.50\left(\mathrm{CHCl}_{3} / i-\mathrm{PrNH}_{2}=9: 1\right)$.

$[\alpha]_{\mathrm{D}}{ }^{24}-12.5\left(c 0.56, \mathrm{CHCl}_{3}\right)$.

FTIR (neat) v 3330,1602, 1541, 1502, 1485, 1457, $1389 \mathrm{~cm}^{-1}$.

${ }^{1} \mathrm{H}$ NMR $\left(400 \mathrm{MHz}, \mathrm{CDCl}_{3}\right) \delta 1.43(1 \mathrm{H}, \mathrm{ddt}, J=12.0,9.5,7.6 \mathrm{~Hz})$, 1.67-1.92 (3H, m), $2.00(4 \mathrm{H}, \mathrm{m}), 2.37(1 \mathrm{H}, \mathrm{br}), 2.72(1 \mathrm{H}, \mathrm{dd}, J=13.2$, $8.0 \mathrm{~Hz}), 2.80-2.84(1 \mathrm{H}, \mathrm{m}), 2.83(1 \mathrm{H}, \mathrm{dd}, J=13.2,5.6 \mathrm{~Hz}), 3.03(1 \mathrm{H}$, ddd, $J=10.0,7.6,5.1 \mathrm{~Hz}), 3.29(4 \mathrm{H}, \mathrm{m}), 3.45(1 \mathrm{H}$, quintet, $J=6.4 \mathrm{~Hz})$, $6.23(1 \mathrm{H}, \mathrm{dd}, J=5.9,2.4 \mathrm{~Hz}), 6.28(1 \mathrm{H}, \mathrm{d}, J=2.4 \mathrm{~Hz}), 8.12(1 \mathrm{H}, \mathrm{d}, J=$ $5.9 \mathrm{~Hz})$.

${ }^{13} \mathrm{C}$ NMR $\left(100 \mathrm{MHz}, \mathrm{CDCl}_{3}\right) \delta 24.87,25.26(\times 2), 31.22,44.80,46.15$, 46.87 (×2), 58.97, 104.89, 106.21, 149.11, 152.16, 159.86.

Anal. Calcd for $\mathrm{C}_{14} \mathrm{H}_{21} \mathrm{~N}_{3} \bullet 0.6 \mathrm{H}_{2} \mathrm{O}: \mathrm{C}, 69.44 ; \mathrm{H}, 9.24 ; \mathrm{N}, 17.35$. Found: C, 69.42; H, 9.40; N, 16.97 .

(S)-3-(Pyrrolidin-2-ylmethyl)pyridine (3d).

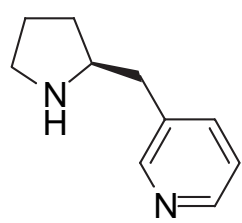

$62 \%$ yield, pale yellow oil; $\mathrm{R}_{\mathrm{f}} 0.29\left(\mathrm{CHCl}_{3} / i-\mathrm{PrNH}_{2}=19: 1\right)$.

$[\alpha]_{\mathrm{D}}{ }^{25}+6.7$ (c 0.60, $\mathrm{CHCl}_{3}$ ).

FTIR (neat) $v 3294,1575,1478,1422,1190,1107,1027,715 \mathrm{~cm}^{-1}$.

${ }^{1} \mathrm{H}$ NMR $\left(400 \mathrm{MHz}, \mathrm{CDCl}_{3}\right) \delta 1.39(1 \mathrm{H}, \mathrm{ddt}, J=11.7,9.5,8.1 \mathrm{~Hz})$, 1.67-1.90 $(4 \mathrm{H}, \mathrm{m}), 2.74(1 \mathrm{H}, \mathrm{d}, J=7.1 \mathrm{~Hz}), 2.85(1 \mathrm{H}, \mathrm{ddd}, J=10.2,8.3$, $6.6 \mathrm{~Hz}), 3.04(1 \mathrm{H}, \mathrm{ddd}, \mathrm{J}=10.2,7.6,5.1 \mathrm{~Hz}), 3.24(1 \mathrm{H}$, quintet, $J=7.1$ $\mathrm{Hz}), 7.22(1 \mathrm{H}, \mathrm{ddd}, J=7.8,4.9,0.7 \mathrm{~Hz}), 7.55(1 \mathrm{H}, \mathrm{ddd}, J=7.8,2.1,1.7$ $\mathrm{Hz}), 8.46(1 \mathrm{H}, \mathrm{dd}, J=4.9,1.7 \mathrm{~Hz}), 8.48(1 \mathrm{H}, \mathrm{d}, J=2.1 \mathrm{~Hz})$.

${ }^{13} \mathrm{C} \mathrm{NMR}\left(100 \mathrm{MHz}, \mathrm{CDCl}_{3}\right) \delta 24.93,31.28,39.67,46.30,60.08,123.25$, $135.51,136.37,147.59,150.30$.

MS $m / z$ (rel intensity) $161\left(\mathrm{M}^{+}-1,2\right), 133$ (4), 118 (4), 93 (51), 70 (100), $65(12)$.

HRMS calcd for $\mathrm{C}_{10} \mathrm{H}_{14} \mathrm{~N}_{2}+\mathrm{H} 163.1235$, found 163.1219 . 


\section{Preparation of $(S)$-2[2-(Pyrrolidin-2-yl)ethyl]pyridine (3e).}

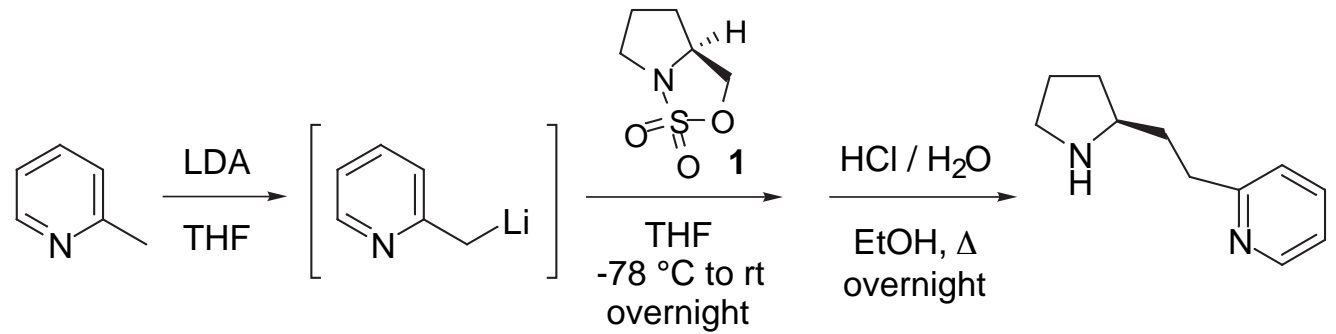

To a solution of LDA $(2.6 \mathrm{mmol})$, prepared from $i-\mathrm{Pr}_{2} \mathrm{NH}(0.37 \mathrm{~mL}$, $2.6 \mathrm{mmol}$ ) and $1.48 \mathrm{M} n$-BuLi in hexane $(17.6 \mathrm{~mL}, 2.6 \mathrm{mmol})$, in dry THF $(8 \mathrm{~mL})$ at $-78{ }^{\circ} \mathrm{C}$ was added dropwise 2-methylpyridine $(0.24 \mathrm{~mL}$, $2.4 \mathrm{mmol}$ ) and the mixture was warmed to $0{ }^{\circ} \mathrm{C}$. After stirring for $1 \mathrm{~h}$, the mixture was recooled to $-78{ }^{\circ} \mathrm{C}$ and a solution of $1(1.37 \mathrm{~g}, 8.4$ mmol) in dry THF $(10 \mathrm{~mL})$ was added dropwise at $-78{ }^{\circ} \mathrm{C}$ and the mixture was allowed to warm to rt (overnight). After evaporation of the solvent, the resulting beige foam was treated with hot $16 \mathrm{~mL}$ of $2 \mathrm{~N} \mathrm{HCl}$ and $16 \mathrm{~mL}$ of EtOH (overnight). The mixture was basified with $50 \%$ $\mathrm{NaOH}$ solution, and extracted with $\mathrm{CH}_{2} \mathrm{Cl}_{2}$. The extracts were dried $\left(\mathrm{MgSO}_{4}\right)$, evaporated, and purified by silica gel column chromatography $\left(\mathrm{CHCl}_{3} / i-\mathrm{PrNH}_{2}=49: 1\right)$ to give the desired product $3 \mathrm{e}(370 \mathrm{mg}, 87 \%)$ as a pale yellow oil.

$\mathrm{R}_{\mathrm{f}} 0.17\left(\mathrm{CHCl}_{3} / i-\mathrm{PrNH}_{2}=19: 1\right)$.

$[\alpha]_{\mathrm{D}}^{25}-4.1\left(c 0.96, \mathrm{CHCl}_{3}\right)$.

FTIR (neat) $v 3388,1592,1568,1475,1435,1404,773,753 \mathrm{~cm}^{-1}$.

${ }^{1} \mathrm{H}$ NMR $\left(400 \mathrm{MHz}, \mathrm{CDCl}_{3}\right) \delta 1.31(1 \mathrm{H}, \mathrm{ddt}, J=12.2,9.5,7.6 \mathrm{~Hz})$, 1.65-1.95 (4H, m), $1.88(2 \mathrm{H}, \mathrm{dt}, J=7.8,6.8 \mathrm{~Hz}), 2.81-2.94(3 \mathrm{H}, \mathrm{m})$, 2.96-3.05 (2H, m), $7.10(1 \mathrm{H}, \mathrm{ddd}, J=7.8,4.9,0.8 \mathrm{~Hz}), 7.17(1 \mathrm{H}, \mathrm{d}, J=$ $7.8 \mathrm{~Hz}), 7.58(1 \mathrm{H}, \mathrm{dt}, J=7.8,2.0 \mathrm{~Hz}), 8.52(1 \mathrm{H}, \mathrm{ddd}, J=4.9,2.0,0.8$ $\mathrm{Hz})$.

${ }^{13} \mathrm{C}$ NMR $\left(100 \mathrm{MHz}, \mathrm{CDCl}_{3}\right) \delta 25.37,31.78,36.28,36.49,46.57,58.75$, $120.90,122.65,136.25,149.14,161.97$.

MS $m / z$ (rel intensity) $175\left(\mathrm{M}^{+}-1,1\right), 106$ (21), 93 (100), 84 (61), 70 
(67).

HRMS calcd for $\mathrm{C}_{11} \mathrm{H}_{16} \mathrm{~N}_{2}-\mathrm{H} 175.1235$, found 175.1234.

Typical Experimental Procedure for the Asymmetric Michael Addition of Cyclohexanone to Nitroolefin.

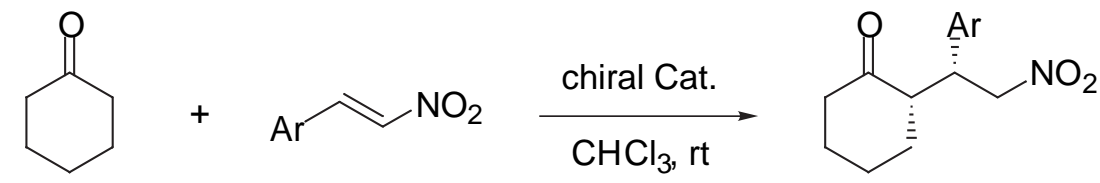

To a mixture of nitroolefin $(0.5 \mathrm{mmol})$ and a chiral pyrrolidinepyridine catalyst $(0.05 \mathrm{mmol})$ in the presence of 2,4dinitrobenzenesulfonic acid monohydrate $(0.025 \mathrm{mmol})$ in dry $\mathrm{CHCl}_{3}$ (freshly distilled from $\left.\mathrm{CaH}_{2}\right)(4 \mathrm{~mL})$ was added cyclohexanone $(1.0 \mathrm{~mL}$, $9.6 \mathrm{mmol})$. The mixture was stirred at $0{ }^{\circ} \mathrm{C}$ until completion of the reaction by monitoring on TLC. The solution was quenched at $0{ }^{\circ} \mathrm{C}$ with $1 \mathrm{~N} \mathrm{HCl}$ and extracted with $\mathrm{CH}_{2} \mathrm{Cl}_{2}$. The combined extracts were dried $\left(\mathrm{MgSO}_{4}\right)$, evaporated, and purified by preparative TLC (Hexane/AcOEt $=4: 1)$ to give the Michael adduct. The ee of the product was determined by chiral HPLC analysis.

Relative (syn) and absolute configuration of the product was determined by comparison with the known ${ }^{1} \mathrm{H}-\mathrm{NMR}$ data and optical rotation values. Compounds $\mathbf{6},{ }^{2} \mathbf{1 0},{ }^{3} \mathbf{1 2},{ }^{4}$ and $\mathbf{1 3}^{5}$ are known.

\section{2-[1-(4-Methoxyphenyl)-2-nitroethyl]cyclohexanone (7).}

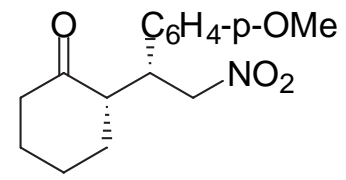

M.p. $148-149{ }^{\circ} \mathrm{C}$ (recryst from $\left.\mathrm{Et}_{2} \mathrm{O}\right) ; \mathrm{R}_{\mathrm{f}} 0.30($ Hexane/AcOEt $=1: 3)$. $[\alpha]_{\mathrm{D}}{ }^{26}-32.0\left(c 0.75, \mathrm{CHCl}_{3}, 99 \%\right.$ ee $)$.

FTIR $(\mathrm{KBr}) \vee 1701,1613,1582,1555,1517 \mathrm{~cm}^{-1}$. 
${ }^{1} \mathrm{H}$ NMR (400 MHz, $\left.\mathrm{CDCl}_{3}\right) \delta$ 1.17-1.28 (1H, m), 1.50-1.84 (4H, m), 2.04-2.11 (1H, m), $2.38(1 \mathrm{H}, \mathrm{ddt}, J=13.0,5.9,1.0 \mathrm{~Hz}), 2.47(1 \mathrm{H}, \mathrm{ddt}, J$ $=13.0,4.0,1.0 \mathrm{~Hz}), 2.65(1 \mathrm{H}, \mathrm{ddt}, J=10.0,5.1,1.0 \mathrm{~Hz}), 3.71(1 \mathrm{H}, \mathrm{dt}, J$ $=10.0,4.6 \mathrm{~Hz}), 3.78(3 \mathrm{H}, \mathrm{s}), 4.58(1 \mathrm{H}, \mathrm{dd}, J=12.2,10.0 \mathrm{~Hz}), 4.91(1 \mathrm{H}$, $\mathrm{dd}, J=12.2,4.6 \mathrm{~Hz}), 6.85\left(2 \mathrm{H}, \mathrm{AA}^{\prime} \mathrm{XX}, J_{\mathrm{AX}}=8.7 \mathrm{~Hz}\right), 7.08(2 \mathrm{H}$, $\left.\mathrm{AA}^{\prime} \mathrm{XX}, J_{\mathrm{AX}}=8.7 \mathrm{~Hz}\right)$.

${ }^{13} \mathrm{C}$ NMR $\left(100 \mathrm{MHz}, \mathrm{CDCl}_{3}\right) \delta 24.98,28.50,33.12,42.70,43.17,52.65$, 55.19, 79.07, 114.27 (×2), $129.14(\times 2), 129.48,158.98,212.06$.

Anal. Calcd for $\mathrm{C}_{15} \mathrm{H}_{19} \mathrm{NO}_{4}: \mathrm{C}, 64.97 ; \mathrm{H}, 6.91 ; \mathrm{N}, 5.05$. Found: $\mathrm{C}$, $65.00 ; \mathrm{H}, 7.25 ; \mathrm{N}, 5.18$.

The ee of the product was determined by chiral HPLC analysis (Chiralpak AD column, $0.46 \times 25 \mathrm{~cm}$, hexane/2-propanol $=80: 20,0.5$ $\left.\mathrm{cm}^{3} / \mathrm{min}\right): \mathrm{R}_{\mathrm{t}}(15.8 \mathrm{~min}) ; 18.6 \mathrm{~min}$.

\section{2-[1-(2-Methoxyphenyl)-2-nitroethyl]cyclohexanone (8).}

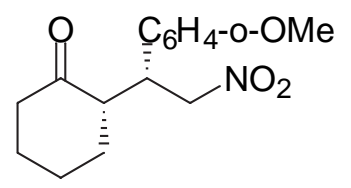

Colorless oil; $\mathrm{R}_{\mathrm{f}} 0.17$ (Hexane/AcOEt $\left.=4: 1\right)$.

$[\alpha]_{\mathrm{D}}{ }^{24}-37.0\left(\mathrm{c} 1.00, \mathrm{CHCl}_{3}, 93 \%\right.$ ee $)$.

FTIR (neat) $v 1705,1600,1586,1550 \mathrm{~cm}^{-1}$.

${ }^{1} \mathrm{H}$ NMR $\left(400 \mathrm{MHz}, \mathrm{CDCl}_{3}\right) \delta 1.20(1 \mathrm{H}, \mathrm{dq}, J=13.2,3.6 \mathrm{~Hz}), 1.57(1 \mathrm{H}$, $\mathrm{tq}, J=12.7,3.6 \mathrm{~Hz}), 1.61-1.80(3 \mathrm{H}, \mathrm{m}), 2.03-2.11(1 \mathrm{H}, \mathrm{m}), 2.38(1 \mathrm{H}, \mathrm{ddt}$, $J=12.2,5.9,1.0 \mathrm{~Hz}), 2.47(1 \mathrm{H}, \mathrm{ddt}, J=13.2,4.6,1.4 \mathrm{~Hz}), 2.98(1 \mathrm{H}, \mathrm{ddt}$, $J=12.0,5.4,1.0 \mathrm{~Hz}), 3.84(3 \mathrm{H}, \mathrm{s}), 3.96(1 \mathrm{H}, \mathrm{ddd}, J=10.5,9.0,5.2 \mathrm{~Hz})$, $4.81(1 \mathrm{H}, \mathrm{dd}, J=12.2,5.2 \mathrm{~Hz}), 4.84(1 \mathrm{H}, \mathrm{dd}, J=12.2,9.0 \mathrm{~Hz}), 6.87(1 \mathrm{H}$, $\mathrm{dd}, J=8.0,1.0 \mathrm{~Hz}), 6.88(1 \mathrm{H}, \mathrm{dt}, J=7.6,1.0 \mathrm{~Hz}), 7.08(1 \mathrm{H}, \mathrm{dd}, J=7.6$, $1.7 \mathrm{~Hz}), 7.24(1 \mathrm{H}, \mathrm{ddd}, J=8.0,7.6,1.7 \mathrm{~Hz})$.

${ }^{13} \mathrm{C}$ NMR $\left(100 \mathrm{MHz}, \mathrm{CDCl}_{3}\right) \delta 25.18,28.57,33.31,41.31,42.74,50.65$, $55.40,77.52,111.04,120.94,125.41,128.95,131.04,157.63,212.54$.

Anal. Calcd for $\mathrm{C}_{15} \mathrm{H}_{19} \mathrm{NO}_{4}: \mathrm{C}, 64.97 ; \mathrm{H}, 6.91 ; \mathrm{N}, 5.05$. Found: $\mathrm{C}$, 
$64.98 ; \mathrm{H}, 7.22 ; \mathrm{N}, 5.18$.

The ee of the product was determined by chiral HPLC analysis (Chiralpak AS column, $0.46 \times 25 \mathrm{~cm}$, hexane/2-propanol $=90: 10,0.5$ $\left.\mathrm{cm}^{3} / \min \right): \mathrm{R}_{\mathrm{t}}(21.8 \mathrm{~min}) ; 24.1 \mathrm{~min}$.

2-(1-Naphthalen-1-yl-2-nitroethyl)cyclohexanone (9).<smiles>O=C1CCCC[C@H]1C[C@H](c1cccc2ccccc12)[N+](=O)[O-]</smiles>

M.p. $133-134{ }^{\circ} \mathrm{C}$ (recryst from $\left.\mathrm{Et}_{2} \mathrm{O}-\mathrm{CH}_{2} \mathrm{Cl}_{2}\right) ; \mathrm{R}_{\mathrm{f}} 0.46($ Hexane/AcOEt $=$ $4: 1)$.

$[\alpha]_{\mathrm{D}}{ }^{25}-46.1$ ( c $1.15, \mathrm{CHCl}_{3}, 99 \%$ ee $)$.

FTIR $(\mathrm{KBr}) \vee 1693,1552,1376 \mathrm{~cm}^{-1}$.

${ }^{1} \mathrm{H}$ NMR $\left(400 \mathrm{MHz}, \mathrm{CDCl}_{3}\right) \delta 1.24(1 \mathrm{H}, \mathrm{dq}, J=12.5,3.2 \mathrm{~Hz}), 1.51(1 \mathrm{H}$, $\mathrm{tq}, J=13.7,3.4 \mathrm{~Hz}), 1.58-1.72(3 \mathrm{H}, \mathrm{m}), 2.04-2.11(1 \mathrm{H}, \mathrm{m}), 2.41(1 \mathrm{H}, \mathrm{ddt}$, $J=12.7,5.9,1.0 \mathrm{~Hz}), 2.50(1 \mathrm{H}, \mathrm{m}), 2.86(1 \mathrm{H}, \mathrm{br}), 4.77(1 \mathrm{H}, \mathrm{br}), 4.90$ $(1 \mathrm{H}, \mathrm{dd}, J=12.7,9.3 \mathrm{~Hz}), 5.07(1 \mathrm{H}, \mathrm{dd}, J=12.7,4.3 \mathrm{~Hz}), 7.37(1 \mathrm{H}, \mathrm{dd}$, $J=7.3,1.2 \mathrm{~Hz}), 7.45(1 \mathrm{H}, \mathrm{dd}, J=8.0,7.3 \mathrm{~Hz}), 7.50(1 \mathrm{H}, \mathrm{ddd}, J=8.0$, $6.8,1.2 \mathrm{~Hz}), 7.55(1 \mathrm{H}, \mathrm{ddd}, \mathrm{J}=8.0,6.8,1.4 \mathrm{~Hz}), 7.77(1 \mathrm{H}, \mathrm{d}, J=8.0 \mathrm{~Hz})$, $7.86(1 \mathrm{H}, \mathrm{d}, J=8.0 \mathrm{~Hz}), 8.16(1 \mathrm{H}, \mathrm{m})$.

${ }^{13} \mathrm{C}$ NMR $\left(100 \mathrm{MHz}, \mathrm{CDCl}_{3}\right) \delta 25.28,28.70,33.29,36.79,42.89,53.79$, $78.67,122.74,123.56,125.34,125.87,126.59,128.17,129.05,132.40$, $133.97,134.59,212.27$.

Anal. Calcd for $\mathrm{C}_{18} \mathrm{H}_{19} \mathrm{NO}_{3}$ : C, 72.71; H, 6.44; N, 4.71. Found: C, $72.53 ; \mathrm{H}, 6.63 ; \mathrm{N}, 4.90$.

The ee of the product was determined by chiral HPLC analysis (Chiralpak AS column, $0.46 \times 25 \mathrm{~cm}$, hexane/2-propanol $=50: 50,0.7$ $\left.\mathrm{cm}^{3} / \min \right): \mathrm{R}_{\mathrm{t}}(9.6 \mathrm{~min}) ; 12.8 \mathrm{~min}$.

2-(2-Nitro-1-thiophen-2-ylethyl)cyclohexanone (11). 


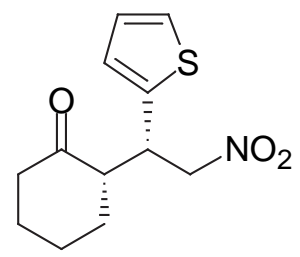

M.p. 82-83 ${ }^{\circ} \mathrm{C}$ (recryst from $\left.\mathrm{Et}_{2} \mathrm{O}\right) ; \mathrm{R}_{\mathrm{f}} 0.46$ (Hexane/AcOEt $=4: 1$ ). $[\alpha]_{\mathrm{D}}{ }^{19}-36.0\left(c 0.91, \mathrm{CHCl}_{3}, 97 \% \mathrm{ee}\right)$.

FTIR $(\mathrm{KBr}) \vee 1699,1554,1445,1433,1384 \mathrm{~cm}^{-1}$.

${ }^{1} \mathrm{H}$ NMR $\left(400 \mathrm{MHz} \mathrm{CDCl}_{3}\right) \delta 1.32(1 \mathrm{H}, \mathrm{dq}, J=13.0,3.6 \mathrm{~Hz}), 1.59-1.73$ $(2 \mathrm{H}, \mathrm{m}), 1.80-1.95(2 \mathrm{H}, \mathrm{m}), 2.06-2.13(1 \mathrm{H}, \mathrm{m}), 2.37(1 \mathrm{H}, \mathrm{ddt}, J=13.0$, 6.1, $1.0 \mathrm{~Hz}), 2.44-2.49(1 \mathrm{H}, \mathrm{m}), 2.68(1 \mathrm{H}, \mathrm{dddd}, J=12.2,8.8,5.1,1.0$ $\mathrm{Hz}), 4.13(1 \mathrm{H}, \mathrm{dt}, J=9.0,4.6 \mathrm{~Hz}), 4.65(1 \mathrm{H}, \mathrm{dd}, J=12.7,9.0 \mathrm{~Hz}), 4.89$ $(1 \mathrm{H}, \mathrm{dd}, J=12.7,4.6 \mathrm{~Hz}), 6.87(1 \mathrm{H}, \mathrm{ddd}, J=3.5,1.2,0.5 \mathrm{~Hz}), 6.93(1 \mathrm{H}$, $\mathrm{dd}, J=5.1,3.5 \mathrm{~Hz}), 7.22(1 \mathrm{H}, \mathrm{ddd}, 5.1,1.2,0.5 \mathrm{~Hz})$.

${ }^{13} \mathrm{C}$ NMR $\left(100 \mathrm{MHz}, \mathrm{CDCl}_{3}\right) \delta 25.08,28.30,32.75,39.41,42.62,53.39$, $79.21,124.97,126.63,126.94,140.52,211.17$.

Anal. Calcd for $\mathrm{C}_{12} \mathrm{H}_{15} \mathrm{NO}_{3} \mathrm{~S}: \mathrm{C}, 56.90 ; \mathrm{H}, 5.97 ; \mathrm{N}, 5.53$. Found: C, $56.99 ; \mathrm{H}, 6.04 ; \mathrm{N}, 5.69$.

The ee of the product was determined by chiral HPLC analysis (Chiralpak AD column, $0.46 \times 25 \mathrm{~cm}$, hexane/2-propanol $=90: 10,0.5$ $\left.\mathrm{cm}^{3} / \min \right): \mathrm{R}_{\mathrm{t}}(19.7 \mathrm{~min}) ; 21.8 \mathrm{~min}$.

\section{References}

1. Cuperly, D.; Gros, P.; Fort, Y. J. Org. Chem. 2002, 67, 238-241.

2. Juaristi, E.; Beck, A. K.; Hansen, J.; Matt, T.; Mukhopadhyay, T.; Simson, M.; Seebach, D. Synthesis 1993, 1271-1290.

3. Blarer, S. J.; Schweizer, W. B.; Seebach, D. Helv. Chim. Acta 1982, 65, 1637-1655.

4. List, B.; Pojarliev, P.; Martin, H. J. Org. Lett. 2001, 3, 2423-2425.

5. Betancort, J. M.; Barbas III, C. F. Org. Lett. 2001, 3, 3737-3740. 